\title{
Serum neutrophil gelatinase-associated lipocalin as an early biomarker of acute kidney injury in subjects undergoing percutaneous transluminal coronary angiogram with normal EGFR
}

\author{
Shylaja.T.V ${ }^{1, *}$, Amit Sonagra² ${ }^{2}$ Asmabi Makandar ${ }^{3}$ Patnaik $^{4}$, Shanthinaidu ${ }^{5}$ \\ ${ }^{1,3}$ Assistant Professor, Dept. of Biochemistry, East Point College of Medical Sciences, Bengaluru, Karnataka, \\ ${ }^{2}$ Assistant Professor, Dept. of Biochemistry, G.M.E.R.S. Medical College, Patan, Gujarat, ${ }^{4}$ Professor, Dept. of \\ Cardiology, Nizam's Institute Of Medical Sciences, Hyderabad, Andhra Pradesh, ${ }^{5}$ Consultant Biochemist and \\ Head of Laboratory Medicine, Care Hospitals, Hyderabad, Andhra Pradesh, India
}

*Corresponding Author:

Email: shylajavishal@gmail.com

\begin{abstract}
Introduction: Detecting AKI in a timely fashion with the current AKIN staging criteria is a challenge because the diagnosis of AKI is usually based on changes in serum creatinine ( $\mathrm{SCr}$ ) which is a poor marker of early renal dysfunction.

Aim: To study the role of Serum Neutrophil Gelatinase-Associated Lipocalcin (NGAL) as early biomarker for the detection of Contrast induced acute kidney injury (CIAKI) in subjects undergoing Percutaneous transluminal coronary angiogram (PTCA) with normal eGFR.

Materials and Methods: Prospective cohort study was conducted, where SCr and serum NGAL were serially measured in a heterogeneous group of subjects $(n=60)$ presenting to cardiology department.

Results: The study population consisted of 60 subjects. All subjects were divided into 2 groups "CIAKI group" and "no-CIAKI group" according to predefined definition. The serum NGAL increased and reached its peak at 4 hours after contrast media (CM) administration and did not returned to baseline by 24 hours while the $\mathrm{SCr}$ increased at 24 hours and reached peak at 48 hours respectively $(P<0.001)$. Thus, 4 hours after $\mathrm{CM}$ administration were considered to be appropriate time point for NGAL measurement and there was no significant correlation between serum NGAL with SCr at 0 hours, 4hours, 24 hours and 48hours.

Conclusion: In our study, we found that serum NGAL promises to be a simple, safe, non-invasive and reliable early biomarker for predicting possible onset of CIAKI following contrast administration.
\end{abstract}

Keywords: Contrast induced acute kidney injury, Percutaneous Trans luminal Coronary angiogram, Serum Creatinine, Estimated glomerular filtration rate.

Received: $05^{\text {th }}$ September, 2017

\section{Introduction}

Acute kidney injury (AKI) Previously referred to as Acute Renal Failure (ARF) is a heterogeneous entity associated with various clinical presentations, treatments, and procedures. The incidence of hospital-acquired AKI varies from $5 \%$ in patients with normal preoperative renal function to $25 \%$ in intensive care unit (ICU) patients. ${ }^{1,2}$ In developed countries,AKI is common in the elderly and hospital-acquired causes dominate, where as in developing countries,AKI is the disease of younger subjects and community-acquired cases are common. Acute diarrheal diseases, acute glomerulonephritis, tropical infections (mainly malaria and leptospirosis), environmental agents and snakebite are the common causes of AKI. ${ }^{3}$ Acute radio contrast nephropathy $(\mathrm{ACN})$ is an important cause of AKI in hospitalized patients undergoing contrast-based procedure. ${ }^{4} \mathrm{SCr}$ has been the predominant marker of renal function in
Accepted: $12^{\text {th }}$ September, 2017

clinical practice for more than half a century and its limitations are well documented. As a marker of renal function rather than injury, the nonlinear relationship between Glomerular Filtration Rate (GFR) and SCr, means GFR may decrease by more than $50 \%$ from normal before a significant rise in $\mathrm{SCr}$ occurs, making $\mathrm{SCr}$ insensitive to small but significant reductions in GFR. ${ }^{5,6} \mathrm{~A}$ particularly acute diagnostic problem is the lack of biochemical markers for detecting early kidney injury that are sensitive and easily applicable in clinical practice and may help to predict the development of AKI.NGAL, as a member of the lipocalin super family,is produced from the nephron in response to tubular epithelial damage. ${ }^{7}$ It has been identified as one of the earliest and potentially one of the most indicative biomarkers of AKI from a diverse array of conditions and can differentiate between prerenal and intrinsic causes. ${ }^{8,9}$ Downstream proteomic analysis also revealed NGAL to be one of the most highly induced protein in the 
kidney after ischemic or nephrotoxic AKI in animal models and studies implicated NGAL as an early diagnostic biomarker for AKI in common clinical situations. ${ }^{10}$ Because of the limitations regarding use of $\mathrm{SCr}$ for the early detection of AKI, and the importance of early detection, this study is being taken up to know the role of serum NGAL that would allow earlier detection of AKI. And it has been conducted in subjects undergoing contrast related procedure as a cause of AKI, since the baseline values were also available in all these subjects.

\section{Materials and Methods}

Study design and patient population: Prospective observational cohort study of 30 cases and 30 controls conducted in the department of Biochemistry, Nizam's Institute of Medical Sciences, Hyderabad, India.

Study material: Subjects admitted in the department of Cardiology to undergo emergency or elective PTCA.

Inclusion criteria: Subjects aged between 18-70 years of either gender who were undergoing elective and emergency PTCA by using Iohexol contrast, with normal SCr levels and normal eGFR $>60 \mathrm{ml} / \mathrm{min} / 1.73 \mathrm{~m}^{2}$ (MDRD formula) admitted to hospital during the study period and willing to participate in this study after obtaining informed consent and ethical committee approval has be taken.

\section{Exclusion criteria:}

1. Subjects with non availability of normal $\mathrm{SCr}$ levels

2. Subjects with non availability of normal eGFR>60 $\mathrm{ml} / \mathrm{min} / 1.73 \mathrm{~m}^{2}$ (MDRD) at admission

3. Subjects underwent contrast related procedure within 1 week or less from the index procedure

Sample Size calculated: Based on baseline data available on serum NGAL the anticipated difference in mean between two groups is 147 and anticipated standard deviation (SD) is 100, to obtain a power of $99 \%$ with type 1 error of 0.001 , the required sample size is 21 . In this study a total of 30 cases and 30 controls are enrolled.

Sample collection: Venous blood samples were drawn from all participants after 12-hour overnight fast before PTCA, and other samples were taken at 4, 24 and 48 hours after the procedure. Blood samples were centrifuged at $2000 \times g$ for 10 minutes, serum separated $\mathrm{SCr}$, and fasting plasma glucose were estimated in overnight fasting sample. And in after procedure samples taken at 4 hours, 24 hours and 48 hours same centrifugation procedure repeated, only $\mathrm{SCr}$ concentrations estimated in all these samples. Remaining serum was stored at $-20^{\circ} \mathrm{C}$.Subjects were observed for primary outcome variable which was the development of CIAKI, defined as an absolute increase in $\mathrm{SCr}$ of $>25 \%$ or $0.5 \mathrm{mg} / \mathrm{dl}$ from baseline values occurring within 24 to 48 hours after the coronary procedure. Finally by looking at the primary outcome.

Cases: 30 Subjects (an increase in $\mathrm{SCr}$ by $>0.5$ $\mathrm{mg} / \mathrm{dl}$ or $>25 \%$ within 3 days after intravascular administration of $\mathrm{CM}$ without an alternative aetiology).

Controls: 30 Subjects (who did not showed $>25 \%$ increase in the SCr at end of 48 hours).

All the data were analysed after completion of study period.

\section{Methods}

1. eGFR is Calculated by applying the MDRD formula, ${ }^{11}$

2. SCr estimated by Jaffe method(IDMStraceable version) $)^{12}$

3. Glucose estimated by HK (hexokinase)method ${ }^{13}$

4. All the parameters were analysed by using fully automated analyzer(Hitachi-912)

5. Serum NGAL levels were measured using a commercially available ELISA kits (BIOPORTO Diagnostics [Gentofte, Denmark]) where serum samples were diluted to 1 in 100 dilution before performing an ELISA assay to fit the concentration of respective NGAL protein in the linear range of the standard curve, the inter and intra assay $\mathrm{CV} \%<10 \%$, the measurement were made in duplicate and in blinded fashion.

\section{Statistical analysis}

Continuous variables were expressed as mean \pm SD and tested with Student's $t$ test and categorical variables were expressed as frequencies and compared with the use of the Pearson chi-square test (Fisher's exact test was applied if the number of observations per cell was fewer than five). The changing trends of serum NGAL was analysed by taking repeated measures at 0 hours, 4 hours, 24 hours and 48 hours before and after procedure. All the analysis was performed using the SPSS 12.0 for Windows.

\section{Results}

Total 60 subjects were included in the present study. All subjects were divided into 2 
groups: "CIAKI group" and "no-CIAKI group" according to predefined definition.

The "no-CIAKI group" included a total of 30 controls, 27 males $(90 \%)$ and 3 females $(10 \%)$ with average age $57.60 \pm 05.4$ yrs [Table 1].The CIAKI group included a total 30 cases, 26 males $(86.7 \%)$ and 4 females $(23.3 \%)$ with average age $60.20 \pm 05.73$ years.

Table 1: The clinical characteristics of the patient population

\begin{tabular}{|l|c|c|c|}
\hline \multicolumn{1}{|c|}{ Variables } & No CIAKI $(\mathbf{n}=\mathbf{3 0})$ & CIAKI $(\mathbf{n}=\mathbf{3 0})$ & $\boldsymbol{P}$ value \\
\hline Demographic Characteristics & & & \\
\hline Mean age & $57.60 \pm 05.4$ & $60.20 \pm 05.73$ & $<0.081$ \\
\hline Male n (\%) & $27(90 \%)$ & $26(86.7 \%)$ & 1.0000 \\
\hline Clinical Characteristics n (\%) & & & \\
\hline Diabetes Mellitus(DM) & $06(20.0 \%)$ & $28(93.3 \%)$ & $<0.0001$ \\
\hline eGFR ml/min/1.73 m2 & $64.90 \pm 03.07$ & $62.80 \pm 02.68$ & 0.0049 \\
\hline $\begin{array}{l}\text { Type of contrast media (CM) } \\
\text { n (\%) }\end{array}$ & & & \\
\hline Iohexol & $30(100 \%)$ & $30(100 \%)$ & - \\
\hline Dose of contrast (ml) & $117.70 \pm 33.70$ & $280.00 \pm 64.17$ & $<0.0001$ \\
\hline
\end{tabular}

Continuous values are expressed as mean $\pm \mathrm{SD}$; categorical values are expressed as total number and percentage of the global population (in parentheses)

As shown in Table 1, that patients with CIAKI group were found no difference in age than controls $60.20 \pm 5.73$ vs $57.60 \pm 05.4$ yrs respectively $(P<0.081)$, DM $(P<0.0001)$, eGFR $(P=0.004)$ compared with patients of "no-CIAKI group" and in subjects who developed CIAKI, a higher dose of CM was administered ( $P<0.0001$ ), while the compared analysis of the patient groups in relation to the molecule type chosen to make coronaries opaque (Iohexol) did not show any significant statistical difference.

Table 2: Changing trends of novel biomarker NGAL after CM exposure in both the subjects $(0$, 4, 24 and 48 hours)

\begin{tabular}{|l|c|c|}
\hline Time (Hours) & $\begin{array}{c}\text { NGAL(ng/ml) } \\
\text { no ClAKI }\end{array}$ & $\begin{array}{c}\text { NGAL(ng/ml) } \\
\text { CIAKI }\end{array}$ \\
\hline 0 & 71.2 & 71.2 \\
\hline 4 & 71.3 & 156.1 \\
\hline 24 & 69.9 & 99.4 \\
\hline 48 & 69.6 & 71.2 \\
\hline
\end{tabular}

Table 3: Changing trends of Conventional marker (SCr) after CM exposure in both the subjects $(0,4,24$ and 48 hours)

\begin{tabular}{|l|c|c|}
\hline Time(Hours) & $\begin{array}{c}\text { S Cr (mmol/L) } \\
\text { No CIAKI }\end{array}$ & $\begin{array}{c}\text { S Cr(mmol/L) } \\
\text { CIAKI }\end{array}$ \\
\hline 0 & 94.7 & 95.3 \\
\hline 4 & 94.0 & 92.7 \\
\hline 24 & 92.7 & 93.6 \\
\hline 48 & 93.0 & 177.0 \\
\hline
\end{tabular}

The changing trends of Serum NGAL and SCr were showed in Table 2, 3 and Fig. 1 \& 2 respectively. The serum NGAL increased and reached its peak at 4 hours after CM administration and did not returned to baseline by 24 hours while the SCr increased at 24 hours and reached peak at 48 hours respectively. Thus, 4 hours after $\mathrm{CM}$ administration were considered to be appropriate time point for NGAL measurement. 
Fig. 1: Shows changing trends of Serum NGAL after CM exposure Changing trends of Serum NGAL after CM exposure

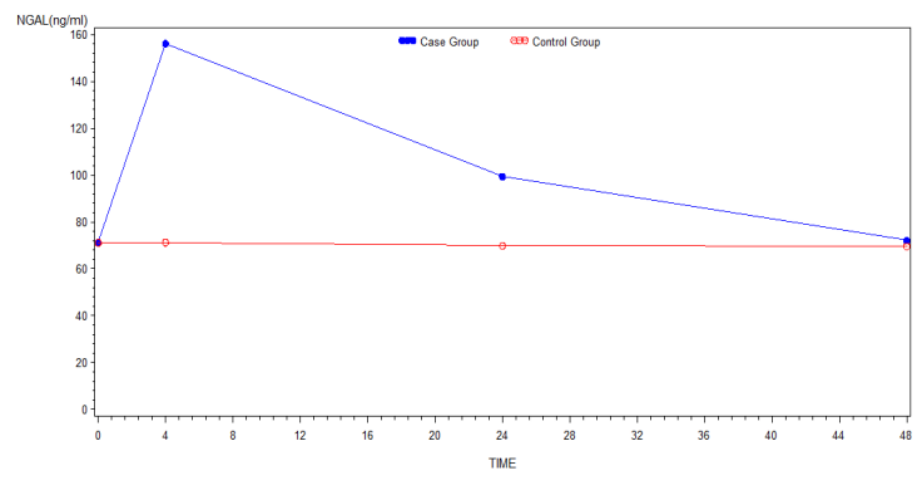

Fig. 2: Shows changing trends of $\mathrm{SCr}$ after CM exposure Changing trends of Creatinine after $\mathrm{CM}$ exposure

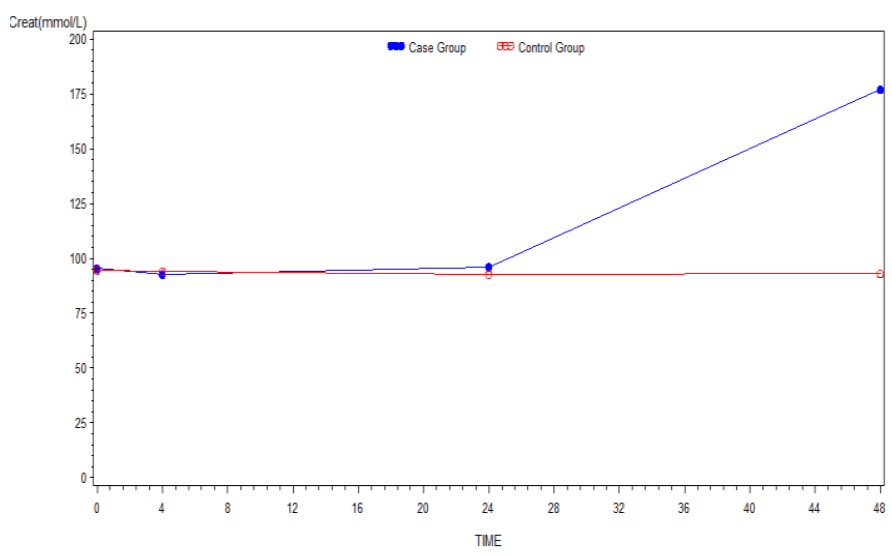

Table 4, CIAKI group showed that, there was a significant increase in mean serum NGAL level after $4 \mathrm{hrs}$ compared to the baseline value before PTCA $(156.07 \pm 24.91 \mathrm{vs} 71.2 \pm 3.14 \mathrm{ng} / \mathrm{ml})$ $(P<0.001)$, where as in the mean SCr level, there was no significant difference between 0,4 , and 24 hours after PTCA but SCr showed significant difference compared to baseline (177 \pm 16.8 vs $94.6 \pm$ $5.7 \mathrm{mmol} / \mathrm{l}(P<0.001)$ at 48 hours

Table 4: Changes in Serum NGAL and SCr before and after PTCA in CIAKI group

\begin{tabular}{|c|c|c|c|c|c|}
\hline Variables & $\begin{array}{c}\text { Before } \\
\text { PTCA }\end{array}$ & $\begin{array}{c}4 \text { hrs after } \\
\text { PTCA }\end{array}$ & $\begin{array}{c}\text { 24 hrs after } \\
\text { PTCA }\end{array}$ & $\begin{array}{c}\mathbf{4 8} \text { hrs after } \\
\text { PTCA }\end{array}$ & P value \\
\hline NGAL(ng/ml) & $71.2 \pm 3.14$ & $156.0 \pm 24.9 \mathrm{a}$ & $99.4 \pm 9.7 \mathrm{~b}$ & $71.1 \pm 4.0$ & $<0.0001 \mathrm{a}, \mathrm{b}$ \\
\hline SCr(mmol/l) & $94.6 \pm 5.7$ & $92.6 \pm 4.4$ & $96.6 \pm 4.3$ & $177 \pm 16.8 \mathrm{c}$ & $<0.0001 \mathrm{c}$ \\
\hline
\end{tabular}

Results expressed as Mean \pm SD

$\mathrm{a}, \mathrm{b}=P<0.0001-$ NGAL Baseline vs $4 \mathrm{hrs}$ and $24 \mathrm{hrs}$ $\mathrm{c}=P<0.0001-\mathrm{SCr}-$ Baseline vs $48 \mathrm{hrs}$

\section{Discussion}

It is important to detect AKI in an initial stage to start early interventions. DM is one of the strongest predictors of AKI after coronary intervention. In our study, we found that occurrence of CIAKI is significantly higher among subjects with DM compared to other subjects. This is in accordance with other study ToprakOet-al. ${ }^{14}$ eGFR found to have significant difference $(P<0.004)$ in CIAKI subjects compared to no CIAKI subjects. Renal function deterioration after exposure to radiographic contrast agents is common in patients with impaired renal function. Volume of contrast showed that the CIAKI risk increases proportionally to the dose of CM like in CIAKI than no CIAKI this is in accordance with other study Kane GC et al. ${ }^{15}$

The discrepancies in the above findings may be due to contributions from several systemic factors like prolonged vasoconstriction, alterations in nitric oxide metabolism that lead to renal vasoconstriction, and impaired auto 
regulation induced by $\mathrm{CM}$ predisposing to medullary hypoxia, in combination with direct cytotoxicity to the renal tubular epithelium. ${ }^{16}$

Numerous studies have demonstrated that the treatment of AKI should be started well before the rise of SCr and immediately after the injury. ${ }^{17-18}$ Sensitive biologic markers of renal tubular injury are needed to detect early AKI because currently AKI diagnosing and staging criteria are entirely based on an increase in $\mathrm{SCr}$ or decrease in urine output. $\mathrm{SCr}$ is insensitive and increases too slow, and urine output is affected by the use of diuretic or prerenal azotemia. In this study, we demonstrated that, the serum NGAL increased and reached its peak at 4 hours after $\mathrm{CM}$ administration and did not returned to baseline by 24 hours, while the $\mathrm{SCr}$ increased at 24 hours and reached peak at 48 hours respectively in subjects undergoing PTCA. Bachorzewska-Gajewska $\mathrm{H}$ et al in their cohort study found a significant rise in serum NGAL after 2 and 4 hrs and a significant rise in urinary NGAL after 4 and 8 hours after coronary angiography, while $\mathrm{SCr}$ and creatinine clearance remained unchanged after procedure. ${ }^{19}$ Similar, but not identical, results were observed by Mishra $\mathbf{J}$ et al who reported a significant rise in serum and urinary NGAL in samples taken after $2 \mathrm{hrs}$ or at the first available sample after cardiopulmonary bypass in children who developed ARF. ${ }^{20}$ Another study by Wagener G et al in patients after cardiac surgery demonstrated higher urinary NGAL in patients developing ARF compared to patients without ARF. ${ }^{21}$ Recent studies have provided clues toward understanding the role of NGAL in the kidney. In the post ischemic kidney, NGAL is markedly up regulated in proximal tubules and distal nephron segments. The similar pattern of proximal tubule NGAL expression was observed following nephrotoxic injury after cisplatin administration. ${ }^{22}$ The reason for NGAL up regulation is not clear, but it may represent a defence mechanism to protect against tubular cell death and preserve renal function, because exogenous NGAL protects against murine ischemia reperfusion injury. ${ }^{23}$ This may be due to marked up regulation of NGAL mRNA and protein levels in the early post-ischemic kidney.

Limitation of our study, since we estimated serum NGAL at 4 hours, so the role of early detection of CIAKI (within 2 hours) in preventing and improving clinical outcomes of high risk patients was not discussed in the current study due to the original study design and limited sample size. Further study with large sample size and intervention may be needed to identify the role of early detection of CIAKI in clinical care of patients with high risk of CIAKI.

\section{Conclusion}

We showed in our study that serum NGAL promises to be a simple, safe, non-invasive and reliable early biomarker for predicting possible onset of CIAKI following contrast administration overriding the conventional renal failure markers.

\section{References}

1. Chertow GM, Lee J, Kuperman GJ, Burdick E, Horsky J, Seger DL, Lee R et al.Guided Medication Dosing For In patients With Renal Insufficiency. Jama 2001;286:2839-44.

2. Liangos O, Wald R, O'bell JW, Price L, Pereira BJ, Jaber BL. Epidemiology And Outcomes Of Acute Renal Failure In Hospitalized Patients: A National Survey. Clin J Am Soc Nephrol 2006;1:43-51.

3. Wang A, Holcslaw T, Bashore TM et al. Exacerbation Of Radio Contrast Nephrotoxicity By Endothelia Receptor Antagonism. Kidney Int 2000;57:1675-80.

4. Schrier RW, Wang W, Poole B, Mitra A. Acute Renal Failure: Definitions, Diagnosis, Pathogenesis And Therapy. J Clin Invest 2004;114:5-14.

5. Han W, Wagener G, Zhu Y, Wang S, Lee H. Urinary Biomarkers In The Early Detection Of Acute Kidney Injury After Cardiac Surgery. Clin J Am Soc Nephrology 2009;4:873-82.

6. Wagener G, Gubitosa G, Wang S, Borregaard N, Kim M, Lee Ht et al. Urinary Neutrophil Gelatinase-Associated Lipocalin And Acute Kidney Injury After Cardiac Surgery. Am J Kidney Dis 2008;52:425-33.

7. Bolignano D, Donato V, Coppolino G, et al. Neutrophil Gelatinase associated Lipocalin (Ngal) As A Marker Of Kidney Damage. Am J Kidney Dis 2008;52:595-605.

8. Wheeler Ds, Devarajan P, Ma Q, et al. Serum Neutrophil Gelatinase-Associated Lipocalin (Ngal) As A Marker Of Acute Kidney Injury In Critically Ill Children With Septic Shock. Crit Care Med 2008;36:1297-303.

9. Seibert FS, Pagonas N, Arndt R, et al. Calprotectin And Neutrophil Gelatinase associated Lipocalin In The Differentiation Of Pre-Renal And Intrinsic Acute Kidney Injury. Acta Physiol (Oxf) 2013;207:700-8.

10. Supavekin $\mathrm{S}$, Zhang W, Kucherlapati RL. Differential Gene Expression Following Early Renal Ischemia-Reperfusion. Kidney Int. 2003;63:1714-24.

11. Poggio E, Wang X, Greene T, Van Lente F, Hall P. Performance Of The Modification Of Diet In Renal Disease And Cockcroft-Gault Equations In The Estimation Of GFR In Health And In Chronic Kidney Disease. J Am Soc Nephrol, 2005;16:459-66.

12. Lamb E, Path F, Price C. Creatinine, Urea and Uric Acid. In: Burtis C, Ashwood E, Bruns D, 
Editors. Teitz Fundamentals of Clinical

Chemistry. 6th Ed. Usa: Elsevier, 2008:P.363-6.

13. Kricka L. Principles of Immunochemical Techniques. In: Teitz Textbook of Clinical Chemistry And Molecular Diagnostics, 4th Edn, Burtis CA, Ashwood ER, Bruns DA (Eds.). New Delhi: Elsevier, 2006. Pp. 219-44.

14. Toprak O, Cirit M, Yesil M. Impact of Diabetic and Prediabetic State on Development Of Contrast-Induced Nephropathy In Patients With Chronic Kidney Disease. Nephrol Dial Transplant. 2007;22:819-26.

15. 15.Kane GC, Doyle BJ, Lerman A, Barsness GW, Best PJ, Rihal CS. et al. Ultra Low Contrast Volumes Reduce Rates Contrast Induced Nephropathy In Patients With Chronic Kidney Disease Undergoing Coronary Angiography. J Am Coll Cardiol. 2008;51:89-90.

16. Wellman TI, Jenkins J, Penar PI, Tranmer B, Zahr R, Lounsbury KM et al. Nitric Oxide And Reactive Oxygen Species Exert Opposing Effects On The Stability Of Hypoxia-Inducible Factor1alpha (Hif-1alpha) In Explants Of Human Pial Arteries. Faseb J 2004;18:379-81.

17. Kelly KJ,Tolkoff-Rubin NE, Rubin RH, William WW, Meehan SM et al. An Oral PlateletActivating Factor Antagonist, Ro-24-4736, Protects the Rat Kidney from Ischemic Injury. Am J Physiol 1996;271: 1061-7.

18. Chiao H, Kohda Y, Mcleroy P, Craig L, Housini I, Star RA: Alpha-Melanocyte-Stimulating Hormone Protects Against Renal Injury After Ischemia In Mice And Rats. J Clin Invest 1997;99:1165-72.

19. Bachorzewska-Gajewska H, Malyszko J, Sitniewska E,Malyszko JS, Dobrzycki S. Neutrophil Gelatinase-Associated Lipocalin And Renal Function After Percutaneous Coronary Angiography. Am J Nephrol 2006;26(3):287-292.

20. Bachorzewska-Gajewska H, Malyszko J, Sitniewska E, Malyszko JS, Dobrzycki S. Neutrophil Gelatinase-Associated Lipocalin (Ngal) Correlations With Cystatin C, Serum Creatinine And Egfr In Patients With Normal Serum Creatinine Undergoing Coronary Angiography. Nephrol Dial Transplant 2007;22:295-6.

21. Wagener G, Jan M and Kim M. Association between Increases In Urinary Neutrophil Gelatinase-Associated Lipocalin And Acute Renal Dysfunction After Adult Cardiac Surgery. Anesthesiology 2006;105:485-91.

22. Mishra J, Mori K, Ma Q, Kelly C, Barasch J, Devarajan P et al. Neutrophil GelatinaseAssociated Lipocalin (NGAL): A Novel Urinary Biomarker For Cisplatin Nephrotoxicity. Am J Nephrol. 2004;24:307-15.

23. Devarajan P, Mishra J, Supavekin S, Patterson Lt, Potter SS. Gene Expression In Early Ischemic Renal Injury Clues Towards Pathogenesis, Biomarker Discovery And Novel Therapeutics. Mol Genet Meta. 2003;80:365-76.

24. Mishra J, Ma Q, Prada A, Mitsnefes M, Zahedi K, Yang $\mathrm{J}$ et al. Identification Of Neutrophil Gelatinase-Associated Lipocalin As A Novel Early Urinary Biomarker For Ischemic Renal Injury. J Am Soc Nephrol 2003;14:2534-43. 